\title{
El punto de partida del análisis bioético de la ciencia y la tecnología: críticas al determinismo*
}

\author{
The starting point of the bioethical analysis \\ of science and technology: criticisms of \\ determinism
}

\section{O ponto de partida da análise bioética da ciência e a tecnologia: críticas ao determinismo}

Fecha de recepción: 29 de agosto de 2016

Fecha de evaluación: 31 de marzo de 2017

Fecha de aceptación: 2 de mayo de 2017

Disponible en línea: 22 de mayo de 2017

Juan Carlos Moreno**

Sergio Néstor Osorio García***

DOI: http://dx.doi.org/10.18359/rlbi.2142

\author{
Cómo citar: \\ Moreno, J. C. y Osorio García, S. N. (2017). El \\ punto de partida del análisis bioético de la ciencia \\ y la tecnología: críticas al determinismo. Revista \\ Latinoamericana de Bioética, 17(2), 76-91. \\ DOI: http://dx.doi.org/10.18359/rlbi.2142
}

* $\quad$ Artículo de reflexión. Producto del Proyecto INV HUM 1804: Riesgo, temor y preocupación. Los imperativos bioéticos de la tecnología en la era planetaria, financiado por la Vicerrectoría de Investigaciones, de la Universidad Militar Nueva Granada.

** Filósofo. Ph.D. en Filosofía; profesor de la Facultad de Educación y Humanidades de la Universidad Militar Nueva Granada; coinvestigador en el proyecto HU1804: Riesgo, temor y preocupación. Los imperativos bioéticos de la tecnología en la era planetaria, financiado por la Vicerrectoría de Investigaciones de la Universidad Militar Nueva Granada, Bogotá, Colombia. Correo electrónico: juan.moreno@unimilitar.edu.co. ORCID: http://orcid. org/0000-0003-4759-4398.

*** Filósofo y teólogo. PhD en Teología; profesor de la Facultad de Educación y Humanidades de la Universidad Militar Nueva Granada; investigador principal del proyecto HU1804: Riesgo, temor y preocupación. Los imperativos bioéticos de la tecnología en la era planetaria, financiado por la Vicerrectoría de Investigaciones de la Universidad Militar Nueva Granada, Bogotá, Colombia. Correo electrónico: sergio.osorio@unimilitar.edu.co. ORCID: http://orcid.org/0000-0003-4759-43980000-0001-5667-7713. 
¿Cuál debería ser el punto de partida del análisis bioético de la ciencia y la tecnología? En casi medio siglo de historia se han formulado diversas perspectivas de análisis bioético de la ciencia y la tecnología. En este artículo se reconoce el amplio contexto teórico sobre el tema y no se va indagar sobre él, sino sobre un asunto más básico: la necesidad de precisar un punto de partida común a los distintos análisis, para evitar algunos presupuestos frecuentemente viciados. En el artículo se propone como punto de partida para el análisis bioético señalado, una perspectiva internalista, orientada fundamentalmente a enfrentar la tarea de desmantelar presupuestos deterministas, y no una mirada externalista, tradicional y moralizante. En el texto se precisan los distintos sentidos de los puntos de vista deterministas sobre la ciencia y la tecnología comúnmente asumidos, y sus relaciones con los juicios morales. También se analiza, de forma comparada, la manera como varios autores y perspectivas toman o cuestionan este tipo de planteamientos. El aporte fundamental del artículo consiste en proponer como perspectiva ética básica, la tarea de abrir la caja negra de los juicios morales, y disolver las precarias comprensiones deterministas de la naturaleza de la ciencia y de la tecnología, en la que se quedan presos muchos de los análisis bioéticos sobre el tema.

Palabras claves: determinismo tecnológico, bioética, internalismo, externalismo, filosofía de la técnica, juicios morales.

Abstract

What should be the starting point of the bioethical analysis of science and technology? In almost half a century of history, different perspectives of bioethical analysis of science and technology have been formulated. This article recognizes the broad theoretical context on the subject and will not inquire about it, but on a more fundamental issue: the need to specify a common starting point for the different analyses, to avoid some frequently flawed budgets. The article proposes as a point of departure for the bioethical analysis indicated an internalist perspective, oriented fundamentally to face the task of dismantling deterministic presuppositions, and not an externalist, traditional and moralizing view. The text specifies the different meanings of the deterministic viewpoints on science and technology commonly assumed, and their relations with moral judgments. It also analyzes, in a comparative form, the way in which several authors and perspectives take or question this type of approach. The fundamental contribution of the article is to propose as a basic ethical perspective the task of opening the black box of moral judgments and dissolving the precarious deterministic understandings of the nature of science and technology, in which many of the bioethical analyses on the subject remain imprisoned.

Keywords: technological determinism, bioethics, internalism, externalism, philosophy of technology.

\section{Resumo}

Qual deve ser o ponto partida da análise bioética da ciência e da tecnologia? Neste artigo propõe-se que a bioética atual deve ter para isso como ponto de partida uma perspectiva internalista, fundamentalmente orientada para enfrentar a tarefa de desmantelar os supostos prévios deterministas, e não um olhar externalista, tradicional e moralizante. No texto precisam-se os diferentes sentidos dos pontos de vista deterministas sobre a ciência e a tecnologia comumente assumidos, e suas relações com os juízos morais. Também se faz uma análise, de forma comparativa, a maneira como diversos autores e perspectivas tomam ou fazem questão neste tipo de abordagem. A principal contribuição do artigo é propor como perspectiva ética básica, a tarefa de abrir a caixa preta dos juízos morais, e dissolver os entendimentos deterministas precários da natureza da ciência e da tecnologia, na qual ficam prisioneiros muitas das análises bioéticas sobre o assunto.

Palavras-chave: determinismo tecnológico, bioética, internalismo, externalismo, filosofia da técnica, julgamentos morais. 


\section{Externalismo ético frente a internalismo ético}

¿Desde qué puntos de vista se han formulado los análisis bioéticos sobre la ciencia y la tecnología? ¿En qué sentido se les ha asignado una carga moral (moralizante) a los conocimientos científicos y a los desarrollos tecnológicos? La evaluación bioética de la ciencia y la tecnología, específicamente los juicios morales sobre las implicaciones y consecuencias de determinado conocimiento científico o de un producto tecnológico, se han formulado tradicionalmente a partir de algunos presupuestos metafísicos y desde perspectivas externas al desarrollo de tales conocimientos y productos. Ciencia y tecnología han sido vistas en sus desarrollos como neutrales, sin agencia, meros medios para las intenciones o las acciones de las personas, o como entidades con una lógica propia o con una configuración interna ajena a los fines humanos, pero con capacidad para generar transformaciones en la vida humana. Ambos casos involucran un punto de partida metafísico sobre la naturaleza de la ciencia y la tecnología, y suponen un lugar externo para el desarrollo de los análisis bioéticos. La responsabilidad de los juicios morales recae en los sujetos que usan o emplean los conocimientos y los productos tecnológicos supuestamente neutros, o en los sujetos que orientan, limitan, encauzan, o le dan un sentido superior a lo que tiene una lógica propia ajena a los fines humanos.

Ambas perspectivas son producto de una concepción cientificista y positivista de la naturaleza y del desarrollo de la ciencia y de la tecnología, y son la base de lo que se ha llamado "determinismo científico-tecnológico".

La bioética surgió con estas concepciones, como una perspectiva capaz de aportar lo que la ciencia y la tecnología no podían ofrecer.
Desde el planteamiento principialista, se vio en la tarea de la bioética un intento de corrección o de limitación a los adelantos científico-técnicos y como una empresa que podría orientarlos y darles sentido ${ }^{1}$. Los presupuestos metafísicos con los cuales se comprendieron la naturaleza de la ciencia y la tecnología, condujeron inicialmente a la bioética a orientar sus análisis morales hacia la posibilidad de trascender la supuesta lógica avalorativa de estas.

Ahora bien, después de casi medio siglo del surgimiento de la bioética, las comprensiones de la ciencia y la tecnología han cambiado profundamente, y no se justifica continuar con el análisis ético de estas desde algunos puntos de vista moralizantes, frecuentes en las décadas pasadas.

Las nuevas miradas sobre la ciencia y la tecnología han relativizado en buena medida el abismo ontológico entre naturaleza y cultura, ciencia, tecnología, sociedad y humanidades. Después de Kuhn, los estudios de la ciencia y la tecnología se han encargado de abrir las cajas negras de la ciencia y la tecnología para poder ver contextos, intereses, valores y procesos sociales, donde solo se veía neutralidad, desarrollo autónomo, lógica positiva inherente.

Más que un faro para orientar la nave perdida, el análisis bioético se ha convertido en una llave para abrir las puertas de lo oculto, lo invisibilizado, o una clave para desencriptar lo que siempre ha estado allí, es decir, la naturaleza humana social de la ciencia y la tecnología. El ocaso de la metafísica conmina a la bioética a convertirse en un saber capaz de leer, de hacer exégesis de todas las intencio-

1 Pensamos que desde la perspectiva de una bioética global, tal y como ha sido planteada por Van Rensselaer Potter, el análisis podría entenderse de otra manera (Potter, 1971). 
nes y fines humanos internos y ocultos configurados en los distintos desarrollos científico-tecnológicos, y no a construir una perspectiva externa que sirva de faro, y finalmente devengue en perspectiva moralizante.

La primera tarea interpretativa que debería emprender la bioética es la del análisis crítico de los presupuestos engañosos, que han sesgado nuestras comprensiones de la ciencia y la tecnología y la han conducido a situar sus pretensiones en un lugar equivocado. Fundamentalmente, la bioética desde las nuevas consideraciones de la ciencia y la tecnología debería empezar por enfrentar la tarea de desmantelar los presupuestos deterministas a partir de los cuales se ha cimentado la idea según la cual, el análisis moral de la ciencia y la tecnología es necesariamente externo y depende de los sujetos y de las sociedades que pueden orientar lo que se concibe como neutro o puede salirse del control.

El determinismo científico-tecnológico es un tema bastante analizado, sobre todo dentro del amplio campo de los estudios de la ciencia y la tecnología. Sin embargo las implicaciones de esta perspectiva para la reflexión bioética al respecto no han sido tenidas en cuenta o han sido muy poco examinadas. La bioética tampoco se ha percatado de las implicaciones que tienen los nuevos estudios sociales sobre la ciencia y la tecnología, específicamente sus posibles aportes para el análisis de los juicos morales. El presente artículo ofrece una contribución a esta importante tarea.

\section{Precisiones sobre el determinismo científico- tecnológico}

Es bastante común y la vez inadvertido suponer un punto de vista determinista en los juicios morales sobre la ciencia y la tecnología, especialmente dentro del contexto de los análisis éticos planteados en los discursos filosóficos, bioéticos, ambientales, etc.

Con frecuencia se encuentran en tales discursos los siguientes planteamientos: "La ciencia y la tecnología, en sus esfuerzos por explicar y transformar la naturaleza, generan riesgos cada vez más peligrosos sobre los sistemas vivos"; "La ciencia y la tecnología, en sus avances vertiginosos, suscitan poderes que ellas no pueden controlar, y se requieren otros conocimientos que las trasciendan para recuperar el sentido y el control"; "La ciencia y la tecnología se desarrollan en el ámbito de una racionalidad afanada por explicar el mundo y por transformarlo, que lamentablemente se ha desligado de otro tipo de racionalidad afanada por comprender el mundo y asegurar nuestra convivencia en él".

Este tipo de planteamientos comparten los siguientes presupuestos:

- Sustancializan la ciencia y la tecnología como conocimientos con lógicas de desarrollo autónomas, que influyen fuertemente sobre la naturaleza y la sociedad.

- Suponen que la ciencia y la tecnología así caracterizadas no tienen el control intrínseco sobre las consecuencias de sus avances,

- Por tanto, es necesario otro ámbito de conocimiento que las trascienda, les dé sentido y pueda garantizar su control.

- La distinción entre estos dos tipos de conocimientos (el científico-tecnológico y el metacientífico) se asienta sobre el supuesto de una dualidad entre dos ámbitos de la razón, del pensamiento o de la vida. 
Estos planteamientos corresponden claramente con lo que se ha denominado "determinismo científico-tecnológico", y también están presentes en muchos de los puntos de vista esgrimidos en los discursos bioéticos sobre las implicaciones de la ciencia y la tecnología en los demás ámbitos de la vida humana. Esta concepción ampliamente extendida la explica Antonio Diéguez (2005) de la siguiente manera:

El discurso sobre el determinismo tecnológico puede hacer referencia a dos cosas que, en principio, son completamente independientes. A veces, sobre todo entre los filósofos, lo que se quiere decir es, como a continuación explicaremos, que la tecnología está sujeta a un proceso autónomo de desarrollo, que, por no obedecer a ningún agente externo a la propia tecnología, se puede considerar como determinado por una lógica interna. Pero entre los historiadores el determinismo tecnológico tiene un significado diferente. Entre ellos se entiende principalmente como la tesis que sostiene que la tecnología determina (o influye de forma decisiva en) el curso de la historia (cfr. Smith y Marx, 1994) (p. 3).

Según el mismo Diéguez, estas versiones de determinismo se mezclan, en un contexto popular, dentro de una concepción más ampliamente sostenida, sintetizada en sus palabras de la siguiente manera:

[El determinismo es] la ausencia de control de la tecnología por parte del ser humano; [...] Según esta interpretación, la sociedad no tiene capacidad para influir en el curso del desarrollo tecnológico. No hay posibilidad real de modificarlo. Estamos abocados a lo que dicte para nosotros la propia tec- nología. Cabe distinguir dos versiones dentro de esta postura: (1) la tecnología (al menos en su forma actual) es intrínsecamente ingobernable y sigue leyes propias (un representante de esta postura sería Jacques Ellul); (2) hemos dejado que las instancias que deberían gobernar y controlar la tecnología no lo hagan (un representante sería Langdon Winner) (p. 5).

Como se hizo notar, esta idea de la ciencia y de la tecnología está profundamente emparentada con la distinción entre dos ámbitos del conocimiento y de la razón humana, planteada en términos kantianos como la distinción entre la razón teórica y la razón práctica. Tal distinción es afín a otra conocida problemática distinción formulada por Snow (1987) entre las dos culturas: la científico-tecnológica y la humanística. La primera está orientada a explicar y controlar la naturaleza, y la segunda a comprender el sentido de la existencia humana.

Estas dos concepciones, el determinismo científico-tecnológico, y la formulación de las dos culturas, han desempeñado un papel de supuestos implícitos negativos que han impedido una comprensión más amplia de la ciencia y la tecnología y de su relación con la sociedad, en cuanto a los retos que enfrentan la ética, la bioética, los estudios ambientales, etc. Ambas concepciones se desprenden de un contexto decimonónico de contraposición entre el humanismo y el cientificismo, o de un entorno que busca colocar como centro el sentido especial de lo humano, y reivindicar la dignidad y la libertad de las personas, no ya frente a la religión, como en el siglo XVI, sino frente a la ciencia y la tecnología.

En líneas generales, de acuerdo con Diéguez (2005), las críticas principales que se 
le hacen a la concepción positivista moderna de la tecnología, emparentada con el cientificismo son las siguientes:

En primer lugar, contempla la tecnología como algo homogéneo, inextricable, sin articulación interna ni niveles diferenciados.

En segundo lugar, convierte a la tesis de la autonomía de la técnica en una tesis cuasi ontológica.

Finalmente, postula unas supuestas leyes de su desarrollo que quedan en la más completa indefinición (p. 6).

De acuerdo con lo dicho, el determinismo entraña una deficiente y precaria concepción de la ciencia y la tecnología, que conduce a una parcial y unidimensional formulación de los juicos morales. Para comprender con más detalle esta problemática relación es preciso hacer algunas aclaraciones y distinciones.

\section{Diferencias entre el determinismo sobre la naturaleza y el determinismo sobre la ciencia y la tecnología}

Aunque comparten sentidos similares, en varios aspectos difiere el punto de vista determinista sobre la ciencia y la tecnología del que han formulado sobre la naturaleza distintas ciencias naturales como la física, la biología, la termodinámica, etc., o varias ciencias sociales como la historia, la sociología y la psicología, entre otras.

Específicamente, en el primer caso los agentes son la ciencia y la tecnología, o sus concepciones. En el segundo, el agente es la naturaleza desde la perspectiva de ciencias, como la física, la biología, la psicología, la sociología, etc. Los determinismos físicos o biológicos, identificados con los puntos de vista de autores como Laplace, Maxwell y otros, y cuestionados por Heisenberg, Poincaré, Prigogine, etc., tienen un sentido ontológico, relacionado con las concepciones sobre la naturaleza, mientras que el determinismo científico-tecnológico juzga en especial las acciones adjudicadas a la ciencia y la tecnología sobre la sociedad, es decir, tiene ante todo un sentido moral en el que se supone, por supuesto, una concepción ontológica sobre la naturaleza de la ciencia y la tecnología. Son puntos de vista deterministas formulados en ámbitos distintos y que no se implican.

En los discursos señalados antes, es usual asumir posiciones críticas frente al determinismo de la naturaleza, pero a la vez suponer un determinismo en relación con los efectos adjudicados a la ciencia y la tecnología sobre la vida humana y la naturaleza. Los cuestionamientos al determinismo sobre la naturaleza no se extienden necesariamente al determinismo sobre el conocimiento de la naturaleza aportados por la ciencia y la tecnología. Por ello, adicional al conocimiento amplio que se tiene hoy sobre el problema del determinismo acerca de la naturaleza, es necesario examinar el punto de vista menos conocido sobre el determinismo en relación con la ciencia y la tecnología.

\section{Análisis de los juicios morales sobre la ciencia y la tecnología}

De manera muy general, en todo juicio moral se evalúa una acción en relación con cuatro aspectos: a) un agente, un sujeto o un responsable de la acción; b) un objeto sobre el que recae la acción; c) unas causas o motivos de la acción (intenciones, propósitos, intereses, inclinaciones, etc), y d) una agencia, o 
unos efectos o consecuencias de la acción sobre un objeto.

El juicio moral es parcial y unidimensional si su formulación se limita en los siguientes sentidos: a) si el agente o sujeto se concibe como el responsable absoluto de la acción, y no se devela a la vez como objeto de otras acciones previas, donde se reproducen o repercuten algunos efectos y consecuencias. En otras palabras, si el agente o sujeto es exclusivamente agente o sujeto, y de ninguna manera es en algún sentido a la vez un objeto que ha sido determinado por otro agente (agente o sujeto absoluto). b) Si el objeto es solo objeto y no es a la vez un sujeto (objeto absoluto). c) Si la causa depende completamente del sujeto o es solo accidental o incidental (responsabilidad absoluta). d) Y si el efecto o la consecuencia se explica solo por una causa (explicación causal o imputabilidad).

Una concepción ética determinista entraña, por tanto, no solo una manera precaria de pensar la ciencia y la tecnología, sino también una formulación parcial del juicio moral en los cuatro sentidos señalados.

Es evidente que el juicio moral en su aplicación debe limitarse o clausurarse en algún sentido. De acuerdo con la eficacia práctica con la que se formula el juicio, no se puede plantear una regresión al infinito de sujetos y de causas de la acción, y por tanto, deben aceptarse algunas cláusulas ceteris paribus en relación con lo que se considera relevante o no de tener en cuenta. Estrictamente, es imposible evitar de manera absoluta algún sentido débil o sutil de determinismo en la manera como debe clausurarse la formulación del juicio. Por tanto, el determinismo del juicio depende del nivel de clausura de este.

Un juicio con un nivel alto de clausura cae bajo un efecto "caja negra"2. Es decir, es un juicio en que el sujeto es solo agente, el objeto es solo objeto, la responsabilidad depende solo del agente y los efectos se explican solo con base en una causa.

En relación con los juicios morales que se formulan sobre la ciencia y la tecnología, los juicios deterministas o "caja negra" son, como dice Diéguez (2005, p. 6), los que las muestran como sujetos homogéneos, autónomos, inextrincables, sin articulación interna, y como reproductores materiales y causantes de las acciones morales. En esos juicios la ciencia y la tecnología se sustancializan y se convierten en entes cuasiontológicos que ejercen su acción sobre una sociedad pasiva; además, muestran como irrelevante la pregunta por su naturaleza -que se supone definida-, y clausuran y limitan la reflexividad acerca de ella y sobre el juicio mismo.

Pero también esos juicios suelen formularse de manera particular en relación con la forma como los conocimientos científicos y los objetos tecnológicos ejercen agencia: en un primer sentido se supone una simple

2 Es interesante extender a los juicios morales el "efecto caja negra" propuesto por los sociólogos para el análisis de la ciencia y la tecnología, pues ello sugiere algo que se invisibiliza y por tanto que se debería desvelar. Latour (2001, p. 363), define el efecto caja negra en relación con la ciencia y la tecnología de la siguiente manera: "Esta es una expresión tomada de la sociología de la ciencia que se refiere al modo en que el trabajo científico y técnico aparece visible como consecuencia de su propio éxito. Cuando una máquina funciona eficazmente, cuando se deja sentado un hecho cualquiera, basta con fijarse únicamente en los datos de entrada y los de salida, es decir, no hace falta fijarse en la complejidad interna del aparato o del hecho". 
forma de agencia extendida ${ }^{3}$, según la cual los conocimientos científicos y los objetos técnicos no son propiamente agentes, pero pueden reproducir la agencia que de alguna forma se les inserta (en términos metafóricos son como agentes zombies). Y en un segundo sentido, en ellos sí se configura una agencia, pero en la medida en que se hace con intenciones ajenas no se les puede adjudicar algún control o responsabilidad sobre esa agencia, aunque a partir de ella se incorporen nuevas causas de acción (también en términos metafóricos son como agentes Frankenstein). En el primer caso se establece una relación causal "ciega", o en la que se señala algún desarrollo científico o algún producto tecnológico como agente, pero se invisibilizan los posibles motivos, intenciones, intereses o los causantes de la acción. La ciencia y la tecnología se conciben como agentes absolutos causantes de las acciones, que no ostentan intenciones, sino que reproducen intenciones. En ellos las intenciones iniciales se materializan, se encarnan y se prolongan o se extienden de forma automática, sin embargo también se invisibilizan una vez que los conocimientos o los productos científico-tecnológicos se desprenden de sus creadores. En el segundo caso, los conocimientos y los productos cobran una dinámica propia autónoma impredecible, amenazante para la sociedad, como el monstruo creado por Frankestein.

En síntesis, los juicios morales que caen bajo el determinismo científico-tecnológico son irreflexivos sobre la ciencia y la tecno-

3 Este concepto lo emplean algunos teóricos de la agencia material de los artefactos técnicos, como Parente (2016, p. 162), y Broncano (2012). Con él dan a entender la forma como la agencia humana se corporiza o encarna en los objetos materiales para el desarrollo eficaz de una acción y para la modulación del entorno, en un nicho o sistema predispuesto para tal fin. logía; las consideran entidades clausuradas y "cajanegrizan" tanto su naturaleza como la formulación del juicio moral mismo. Las conciben como agentes materiales no responsables de las acciones, o como agentes autónomos que se salen de control. En ambos casos se clausura su análisis.

\section{El problema de la agencia}

La asignación de algún tipo de responsabilidad a la ciencia y a la tecnología involucra algunos supuestos muy problemáticos que se deben explicitar.

En primer lugar, supone un punto de vista muy superficial tomar como agentes de manera general a "la ciencia" y "la tecnología", pues estos son conceptos abstractos universales de los que difícilmente se puede predicar algo. Es mucho más concreta la referencia a $\mathrm{x}$ conocimiento científico, o $\mathrm{x}$ proceso o sistema tecnológico. Este tipo de generalizaciones se formulan por economía del lenguaje, o son frecuentes en algunos discursos generales con propósitos sociales amplios, por ejemplo, en los planes de desarrollo, o en los discursos filosóficos que plantean una reflexión general abstracta. Por lo tanto, los juicios en los que de manera general se responsabiliza a la ciencia y a la tecnología de algo son vacíos, pues involucran un agente abstracto, o un concepto universal que no existe de forma concreta en ninguna parte. Además, caen en la falacia de "la causa falsa", al establecer como causa de las acciones algo que se asocia como responsable porque lo precede.

El problema fundamental que se pone en juego en los juicios moralizantes analizados con anterioridad, es el de la asignación de agencia o de responsabilidad a entidades materiales no intencionales como los conocimientos científicos y los productos o procesos 
tecnológicos. ¿Cómo pueden ser ellos agentes responsables de alguna acción? Desde el punto de vista más común se supone que los conocimientos y los productos o procesos tecnológicos son cosas neutras no responsables de nada, y la responsabilidad moral le corresponde a los sujetos humanos que los emplean; no hay lugar para un determinismo científico o tecnológico, pero tampoco tiene sentido un análisis moral de la ciencia y la tecnología, sino de los sujetos y sociedades que las hacen y las usan.

Sin embargo para algunos autores recientes como Latour (1998a), Hacking (1996), Winner (1986), Pickering (1995) y muchos otros dentro del campo de los estudios sociales de la ciencia y la tecnología, los conocimientos científicos concretos y los productos y procesos tecnológicos no deben pensarse como entes materiales neutros, sino como agentes materiales en los que de alguna forma se reproducen responsabilidades.

En líneas generales Latour (1998a), Hacking (1996) y Pickering (1995) muestran que lo material tiene efectivamente agencia. Pero en términos morales concretos, ¿cómo se puede predicar de ellas alguna responsabilidad moral?, y ¿cómo se puede adjudicar algún tipo de responsabilidad sin caer en un punto de vista determinista?

La respuesta que ofrece Landong Winner (1986) es que los sistemas tecnológicos pueden contener alguna intención moral o política en dos formas: encarnada o inherente. La primera es cuando se configura la materialidad del sistema para que cumpla de manera eficaz un propósito específico, como en su ejemplo famoso de los puentes racistas de Long Island, en Nueva York. Y la intención inherente se manifiesta en algunos sistemas tecnológicos que implican riesgos inelu- dibles, y sobre los que la sociedad exige el cumplimiento de unas normas para su desarrollo. Por ejemplo, la producción de materiales radioactivos, o de alimentos transgénicos, o la producción y consumo de licores. En estos casos los productos tecnológicos tienen un sentido moral y político inherente.

Este planteamiento de Winner (1986) es muy concreto e interesante, pero la forma encarnada o inherente como plantea que los sistemas tecnológicos son depositarios de intenciones no puede evitar cierto punto de vista determinista tácito.

El construccionismo científico-tecnológico representa un punto de vista equivalente, pero que evita sustancializar de alguna manera los desarrollos de la ciencia y la tecnología. Para esta perspectiva los desarrollos científicos y tecnológicos no son depositarios de intenciones, sino que son sistemas sociales materializados. O son formas eficaces de materializar e invisibilizar intenciones. Como dice Latour (1998b) "la tecnología es la sociedad hecha para que dure". La tecnología no solo representa, encarna, manifiesta, reproduce las intenciones sociales, sino que ella es social. Los sistemas tecnológicos son relaciones e intenciones sociales cosificadas.

El problema de este punto de vista es que desplaza la formulación implícita de un determinismo científico-tecnológico hacia la afirmación implícita de un determinismo social.

Para intentar salir de la oscilación entre estos dos determinismos, se han propuesto conceptos como el de momentum, planteado por Thomas Hughes (1994). De acuerdo con este concepto, las tecnologías conservan y movilizan por un tiempo limitado las intenciones. Por más que ellas se configuren con 
una idea específica, los contextos y los usos cambian, y las intenciones se debilitan, desaparecen o se transforman.

En fin, el problema de la manera como las tecnologías y los conocimientos agencian intenciones es complejo y requiere de un análisis extenso en otro texto.

Hasta aquí hemos estudiado las modalidades, los supuestos y las implicaciones del determinismo científico-tecnológico y sus relaciones con los juicios morales que lo asumen. Enseguida, vamos a mostrar cómo algunas perspectivas teóricas de estudio de la ciencia y la tecnología cuestionan puntos de vista deterministas, y lo que ello implica para el análisis bioético.

\section{Juicios morales deterministas sobre la ciencia y la tecnología en las interpretaciones de algunos filósofos de la técnica}

El determinismo científico-tecnológico se ha manifiestado con frecuencia con diferentes grados y matices en los juicos morales de algunos discursos éticos y bioéticos que han analizado el devenir de la ciencia y de la tecnología, y sus efectos sobre la vida humana, a partir especialmente de la interpretación de conocidos planteamientos filosóficos clásicos sobre la técnica, por ejemplo en los juicios en los que se le responsabiliza de algunos males morales a partir de la interpretación de la crítica de Marx sobre su devenir como una forma de apropiación instrumental de la naturaleza, o como un sistema de explotación de los recursos y de la fuerza de trabajo que enajena al ser humano.

O desde una cierta interpretación heideggeriana, en la que se inculpa de manera general a la técnica como un proceso que conmi- na a la naturaleza a liberar sus recursos, de manera ilimitada, o como manifestación de un modo inauténtico de lo existente. $O$ de ser un medio que se implementa con miras a un fin, pero que amenaza con escapar al control del hombre y convertirse en un fin en sí mismo.

O a partir de las interpretaciones que se hacen de autores como Ellul (1964) y Marcuse (1993), en los juicios que advierten cómo la técnica moderna se sale del control del ser humano y se vuelve autónoma, imponiendo su propia ley. La técnica impone racionalidad orientada hacia la eficacia en todas las demás esferas de la actividad humana tecnificando crecientemente al ser humano y a su entorno. La técnica se concibe como un agente material o un conjunto de procesos sin sujetos intencionales, de naturaleza totalitaria, que invade y domina a la humanidad (Vinck, 2012). O que conduce al "autoritarismo técnico", como lo planteó Mumford (1964), es decir, a la situación en la cual la vida humana se pone en función del desarrollo técnico.

Sería una labor que escapa a los propósitos de este artículo aclarar en qué sentidos los juicios deterministas relacionados con estos autores clásicos mencionados se deben adjudicar a los planteamientos de los autores o surgen de interpretaciones sesgadas. Y también el tipo de determinismo que se encuentra implícito en los juicios. Pero por lo pronto valgan de ejemplo.

\section{Límites al determinismo dentro de la filosofía de la técnica}

Como se planteó, los juicios morales deterministas sobre la ciencia y la tecnología surgen a partir de la reducida y simplificacionista concepción de la agencia adjudicada a la ciencia y la tecnología sobre la sociedad y 
la vida humana. Ese tipo de idea se ha visto cuestionada y desarticulada en algunos estudios más complejos y profundos, producidos desde varias perspectivas teóricas, inicialmente a partir de una reflexión sobre la naturaleza de la ciencia y la tecnología.

Específicamente, para algunos filósofos de comienzos del siglo XX, como Mumford, Heidegger y Simondon (cfr. Vinck 2012, pp. 17-37) la técnica es consubstancial y constitutiva del ser humano, y en esa medida no es un agente externo que determina su vida. Aún más, para el filósofo contemporáneo Bernard Stiegler (2002a), la técnica es la condición esencial material temporal de la existencia humana.

En las concepciones de estos autores, se diluyen buena parte de los juicios deterministas sobre la técnica, en la medida en que no la substraen ni sustancializan fuera de la naturaleza humana. La técnica no es considerada como un agente externo al ser humano, homogéneo, autónomo, sin articulación interna. O simplemente como un agente reproductor material de las acciones morales. En estos autores la técnica se piensa como un elemento constituyente de la compleja naturaleza humana, no como un agente particular que ejerce su acción sobre la sociedad pasiva.

En concreto, para Mumford (1964) la técnica y la sociedad se codeterminan. Según este autor, existe una coevolución global entre la técnica y la sociedad. La técnica prolonga y refuerza el desarrollo de la sociedad, y la sociedad prolonga y refuerza el desarrollo de la técnica. La máquina es la materialización de la megamáquina política (Vinck, 2012). Y la sociedad humana que le da preponderancia al desarrollo técnico, impone de forma autoritaria una manera política de devenir técnico y social.

Desde el mismo ámbito de la reflexión ontológica planteada por Heidegger (1994), para Simondon (2014) no existe ningún sistema científico-tecnológico que pueda ser comprendido de manera individual. Por tanto, no existe una brecha ontológica entre la técnica y la sociedad, sino diferentes procesos de individuación en los que la técnica cumple una función esencial. Para él las técnicas son la culminación de un proceso, de una génesis del "esfuerzo humano", en la que hombre y máquina son mediadores recíprocos. La técnica forma parte en sentido ontológico del mundo humano, en ella se objetiva la relación con el mundo.

Por la misma senda planteada por Heidegger y Simondon, el filósofo contemporáneo Bernard Stiegler (2002 a, b y 2004) emprende una reflexión de carácter antropológico y ontológico, más ambiciosa que la de los autores mencionados, en donde la técnica es central. Para él la técnica es la forma fundamental de autoconstitución del único ser que no goza de una naturaleza predefinida específica, o que define en su existencia su propia naturaleza. La técnica es constituyente del ser humano, este es a la vez constituyente de la técnica.

Más que una teoría de la técnica, este autor propone una ontología y una antropología en la que la técnica cumple un papel central como substrato material y temporal de la existencia humana. Frente al desconocimiento que ha tenido la filosofía de la materialidad del pensamiento y del conocimiento, la técnica significa la materialidad sobre la que reposa el pensamiento, o el ya-ahí material temporal de la existencia. En términos ontológicos, en la técnica se ha conformado 
el sentido temporal material de la existencia humana, pues a través de ella se ha constituido la memoria filogenética que ha posibilitado la existencia humana.

Esta comprensión de la técnica como sedimento temporal de la existencia, o como memoria material, es un aporte fundamental de Stiegler. Ni siquiera Marx -el más grande pensador de la industria- o Heidegger consideraron la técnica en este sentido (cfr. Sei, 2004, p. 347).

Frente al olvido de la técnica en la filosofía, Stiegler propone que, "sin sustratos materiales, sin objetos técnicos que funjan como dispositivos retentivos, el intelecto y la razón del sujeto no serían más que vapores" (Sei, 2004, p. 347).

Este carácter retentivo, temporal y material que se da en la existencia humana a través de la técnica, se encuentra explícito también en la concepción de Bruno Latour (1998b) cuando plantea: "la técnica es la sociedad hecha para que dure". Stricto sensu, este planteamiento no solo es un análisis ontológico de la técnica, sino de manera más amplia, un análisis material de la ontología. Stiegler propone un desplazamiento del logos hacia el tecno-logos, en la forma como se entienden los procesos de individuación en la tradición hermenéutica que recoge. Él analiza la individuación no solo con base en el lenguaje, sino fundamentalmente por la "condición instrumental o técnica" del ser humano. (Cfr. Sei, 2004, p. 348).

Desde este punto de vista, el análisis ontológico de la técnica ofrecido por Stiegler, es más amplio que el propuesto por Heidegger. De acuerdo con Mario Sei (2004),

Stiegler, en efecto, no cuestiona la estructura fundamental de la hermenéutica fe- nomenológica, el hecho -es decir- de considerar que toda individuación reposa sobre un fondo pre-individual re-interpretado y re-actualizado, pero desplaza el fulcro de esa estructura desde el tema del lenguaje al tema de lo que podríamos denominar la "condición instrumental" (p. 348).

A pesar del sentido ontológico con el que Heidegger comprendió la técnica, él no alcanzó a advertir que ella es la condición esencial del tiempo. Es decir, que el ya-ahí dispuesto por la técnica constituye la condición esencial del sentido anticipatorio del Dasein. "La desconfianza con la que Heidegger afronta el cálculo, la medida y la fijación en general, entendidos como voluntad de determinar lo indeterminado, olvida que ese programa es la condición misma de toda temporalización" (Sei, 2004, p. 351).

\section{La naturaleza social de la técnica}

Los planteamientos filosóficos que hemos expuesto, diluyen la tendencia a clausurar los juicios deterministas sobre la ciencia y la tecnología, en la medida en que indagan sobre la naturaleza de ellas, o mientras analizan la ciencia y la tecnología como agentes complejos, en sentido ontológico y antropológico.

Sin embargo tienen poca eficacia práctica en tanto recursos o marcos conceptuales útiles para analizar las formas como en la vida ordinaria los conocimientos y los procesos y productos tecnológicos median las acciones humanas. Este tipo de analítica existencial filosófica sobre la ciencia y la tecnología es general y abstracta, y traza un camino muy largo para pasar del discurso y de la reflexión, al análisis de los problemas ordinarios y concretos de la ciencia y la tecnología. 
Un análisis mucho más concreto sobre la naturaleza de la ciencia y la tecnología se hace en el construccionismo social de la tecnología, propuesto especialmente por Bijker, Hughes y Pinch (1987) en su famoso texto The Social Construction of Technological Systems.

En continuidad con los planteamientos construccionistas de la escuela de Edimburgo, estos autores comprenden la tecnología como un constructo social. Ella carece de autonomía, de esencia y se explica por la sociedad. Aunque esa configuración social se suele invisibilizar después de su estabilización y se tiende a considerar la tecnología independiente de la sociedad.

Inicialmente, antes de su estabilización, o de la asimilación social de un paradigma, los cambios tecnológicos se mantienen "abiertos" a las determinaciones sociales. Se conserva, de acuerdo con los construccionistas de la escuela de Edimburgo, una "flexibilidad interpretativa" (Bijker, Hughes, Pinch, 1987). Pero después de configurarse de manera estable el conjunto de intereses, ejercicios de poder, representaciones, evaluaciones, etc., en juego, a favor o en contra de las distintas opciones sociotécnicas, se produce un proceso de "clausura", o de "cajanegrización", que invisibiliza las causas sociales determinantes en la emergencia del cambio tecnológico. De esta forma, un proceso sociotécnico clausurado, de acuerdo con Collins (1981), se mantiene como "no controversial", y por lo tanto, para comprender el proceso de su construcción social se hace necesario abrir la controversia.

Según esta perspectiva, la sociedad proyecta su fuerza en la tecnología, se transforma a sí misma a través de ella. La tecnología es un tipo de conocimiento y de acciones que materializan las intenciones de la socie- dad; es un vector de influencia de la sociedad sobre sí misma, es la sociedad hecha para que dure (Latour, 1998b).

Este punto de vista tiene un gran poder explicativo, pues permite vincular el desarrollo de los conocimientos científicos y de los sistemas tecnológicos a situaciones, contextos, intereses e intenciones concretas. Hace posible una reflexión sobre la naturaleza social de la ciencia y la tecnología, en contraste con la reflexión general y abstracta de los enfoques filosóficos antes mencionados, y en esa medida, al igual que esos enfoques, limita el planteamiento del determinismo científico y tecnológico. Pero esa capacidad para ofrecer explicaciones viene con el costo de inclinar la balanza hacia otro tipo de determinismo, el social. Por tanto, podríamos buscar un punto intermedio que nos permita acoger lo mejor de los puntos de vista señalados.

\section{El tejido sin costuras}

Frente a esta posible polarización entre dos determinismos (el científico-tecnológico y el social), otros enfoques más recientes como el sistémico y el conocido como "actor-red", buscan explicar las interacciones complejas, no deterministas, en las que se interdefinen tanto la tecnología como la sociedad. Para el enfoque sistémico propuesto por Hughes (1983), y el enfoque actor-red de Latour, Callon y Law (Latour, 1992), la desarticulación del determinismo tecnológico no supone como consecuencia la inclinación hacia un determinismo social.

De forma similar a los demás enfoques expuestos, para estas perspectivas no existe una esencia escondida o una lógica implacable de los sistemas científico-técnicos. Pero tampoco la tecnología es una proyec- 
ción ciega de la sociedad. Estas perspectivas vuelven a considerar que los sistemas tecnológicos impactan de alguna forma a la sociedad, y a la vez los procesos sociales construyen las tecnologías, pero no como lo plantea una concepción determinista, considerándolos como entidades ontológicas con algún tipo de autonomía, o a la sociedad como la base de todo.

Para evitar caer en lo anterior, estas perspectivas profundizan en dos condiciones fundamentales de los sistemas sociotécnicos. Por un lado en su condición continua, fluida, híbrida, sin separaciones o costuras, y por otro lado, en su condición dinámica, activa. En relación con la primera, conciben que la realidad sociotécnica es consecuencia de múltiples asociaciones y no hay nada misterioso por ser develado. Asociaciones heterogéneas de acciones humanas y agencias materiales, entretejen procesos de transformación (traducciones), para configurar un ensamble, una red sociotécnica híbrida, fluida, continua, como la famosa expresión de Latour de un tejido sin costuras (Latour, 1992).

Y en relación con la segunda condición, consideran la ciencia y la tecnología no solo como conjunto de representaciones y de productos, sino especialmente como proceso de acciones modeladoras del conocimiento, la realidad y la sociedad (Latour, 1992), en correspondencia con la concepción alternativa de la ciencia como acción (Moreno, 2014), ignorada en las tradicionales historias de la ciencia. Desde tal perspectiva, emplean especialmente el concepto de momentum propuesto por Hughes (1994), como una especie de inercia de los sistemas sociotécnicos, en que unas tecnologías y unos procesos sociales conducen o dan lugar a otras tecnologías y a otros procesos sociales, con diferentes tipos de interacciones, en los que fluyen las asociaciones y se hacen complejas las configuraciones, intenciones y orientaciones iniciales.

Este enfoque se aparta tanto del determinismo tecnológico como del social. Específicamente, el cambio tecnológico es endógeno a la sociedad y refleja un estado de esta, fuera de un punto de vista determinista sea metafísico o social. Y a diferencia de todos los enfoques anteriores, le otorga, sin asumir una perspectiva determinista tecnológica, "agencia" a los artefactos (considerados como "actantes" o "agentes no-humanos"), y no solo a los actores humanos. Este punto de vista se conoce como el principio de "simetría general o radical", en relación con el principio de simetría de la Sociology of Scientific Knowledge, pues implica una ampliación de las condiciones de simetría en las explicaciones, en relación con lo que Latour (1998a) denomina "la mediación técnica". Esta integración de agencia humana y no humana se encuentra implícita en el concepto de "agenciamiento", aportado previamente por Deleuze (2002).

Las transformaciones que se dan a través de los agenciamientos se llaman traducciones (Latour, 1992). Ellas se comprenden a través de diferentes subprocesos en diferentes ámbitos. Específicamente en las relaciones sociedad-técnica, las traducciones implican los procesos de problematización, interés, enrolamiento y movilizaciones. En las relaciones entre lo material y lo humano implica composición y delegación. En la relación lenguaje-realidad abarcan los procesos de inscripción.

Respecto a los demás enfoques, esta perspectiva aporta una concepción más integradora, simétrica, dinámica, fluida y continua de los cambios sociotécnicos, que elude las 
distintas formas de determinismos. En términos metafóricos, las interacciones entre la ciencia, la tecnología y la sociedad las piensa como una red o "un tejido sin costuras", en el cual los sistemas son indisociablemente técnicos y sociales. Solo hay redes sociotécnicas y ensambles heterogéneos.

\section{Conclusiones}

En líneas generales, a través de los planteamientos desarrollados en este artículo hemos pretendido justificar la necesidad e importancia de examinar los supuestos deterministas de los discursos que proponen a la ciencia y la tecnología como agentes autónomos sobre la sociedad.

Bioéticamente, es fundamental examinar los supuestos deterministas que existen sobre la ciencia y la técnica para evitar la común parcialidad y simplicidad con la que se formulan los juicios morales sobre ellas. Esta parcialidad se sostiene sobre todo por la irreflexiva tendencia a detenerse en lo que posiblemente ocasionan las acciones, basada en un prejuiciado y pobre análisis de las posibles causas de las acciones. Es decir, un defecto común de los juicios morales mencionados es el de concentrar el análisis en el señalamiento de todos los posibles males y problemas vinculados con el avance de la ciencia y la tecnología, mientras se ignora y deja de lado la forma como estas se representan como actores responsables.

En su afán de eficacia práctica, los juicios "cajanegrizan" toda la complejidad que entraña las adjudicaciones de responsabilidad a aquello que se señala de manera tan general en los conceptos de ciencia y tecnología. En sus críticas al cientificismo y a la tecnología, algunas posiciones bioéticas muy empleadas, sobre todo las posiciones principialistas, estigmatizan y sustancializan la misma imagen que critican. De esta forma, tanto los cientificistas, como los bioeticistas que asumen un punto de vista anticientificista, comparten la misma concepción problemática de la ciencia y la tecnología, pero desde polos opuestos.

Una nueva pretensión de la bioética global tiene que ser el análisis de todo lo que implica abrir esa caja negra de los juicios morales deterministas. Para ello, podría comenzar con hacer explícitos los distintos supuestos involucrados en los puntos de vista deterministas sobre la ciencia y la tecnología, valiéndose para ello de los estudios que problematizan y cuestionan la visión reduccionista de estas.

El punto de partida propuesto en este artículo para el desarrollo de la bioética, representa una nueva perspectiva internalista, global y posmetafísica, apoyada en los estudios de la ciencia y la tecnología.

\section{Referencias}

Bijker, W., Hughes, T. y Pinch, T. (Eds.) (1987). The Social Construction of Technological Systems: New Directions in the Sociology and History of Technology. Cambridge y Londres: The MIT Press.

Broncano, F. (2012). La estrategia del simbionte. Cultura material para nuevas humanidades. Salamanca: Delirio.

Collins, H. M. (Ed.) (1981). Knowledge and Controversy: Studies of Modern Natural Science. Special issue of Social Studies of Science, 11(1).

Deleuze, G. (2002), Diferencia y repetición. Buenos Aires: Amorrortu.

Diéguez, A. (2005). El determinismo tecnológico: Indicaciones para su interpretación. Argumentos de Razón Técnica, 8, 67-87. 
Ellul, J. (1964). The technological society. Nueva York: Knopf.

Hacking, I. (1996). Representar e intervenir (Trad. S. Martínez).México, D. F.: Paidós.

Heidegger, M. (1994). La pregunta por la técnica y ciencia y meditación, En Conferencias y artículos (pp. 9-37), Eustaquio Barjau (Trad.). Barcelona: Ediciones del Serbal.

Hughes, T. (1983). Networks of Power Electrification in Western Society, 1880-1930. Londres: Johns Hopkins University Press.

Hughes, T. (1994). Technological momentum. En M. Smith y L. Marx (Eds.) Does Technology Drive History?: The Dilemma of Technological Determinism (pp. 101-113). Cambridge y Londres: MIT Press.

Latour, B. (1992). Ciencia en acción: cómo seguir a los científicos e ingenieros a través de la sociedad. Barcelona: Labor.

Latour, B. (1998a). De la mediación técnica: filosofía, sociología, genealogía. En M. Domenech y F. Tirado (Comps.), Sociología simétrica. Ensayos sobre ciencia, tecnología y sociedad (pp. 249-302). Barcelona: Gedisa.

Latour, B. (1998b). La tecnología es la sociedad hecha para que dure. En M. Domènech y F. Tirado (Comps.), Sociología simétrica. Ensayos sobre ciencia, tecnología y sociedad (pp. 109-142). Barcelona: Gedisa.

Latour, B. (2001). La esperanza de Pandora. Fernández T. (Trad.). Barcelona: Gedisa.

Marcuse, H. (1993). El hombre unidimensional. Buenos Aires: Planeta-Agostini.

Moreno, J. C. (2014). La ciencia como acción. Universitas Philosophica, 31(63), 289-301.

Mumford, L. (1964). Authoritarian and Democratic Technics, Technology and Culture, 5, 1-8. DOI: https://doi.org/10.2307/3101118
Parente, D. (2016). Los artefactos en cuanto posibilitadores de acción. Problemas en torno a la noción de agencia material en el debate contemporáneo. Revista Colombina de Filosofía de la Ciencia, 16(33), 139-168.

Pickering, A. (1995). The Mangle of Practice. Time, Agency, and Science. Chicago: The University of Chicago Press. DOI: https://doi.org/10.7208/chicago/9780226668253.001.0001

Potter, V. R. (1971). Bioethics: Bridge to the Future. Nueva Jersey: Prentice Hall.

Sei, M. (2004). Técnica, memoria e individuación: la perspectiva de Bernard Stiegler. Logos. Anales del Seminario de Metafísica, 37, 337-363.

Simondon, G. (2014). La individuación a la luz de las nociones de forma y de información. Buenos Aires: Editorial Captus.

Smith, M. R. y Marx, L. (Eds.) (1994). Does Technology Drive History? The Dilemma of Technological Determinism. Cambridge y Londres: MIT Press.

Snow, C. P. (1987) Las dos culturas y un segundo enfoque. Madrid: Alianza.

Stiegler, B. (2002a). La técnica y el tiempo I. El pecado de Epimeteo (Trad. B. Morales). Hondarribia: Hirú.

Stiegler, B. (2002b). La técnica y el tiempo II. La desorientación (Trad. B. Morales). Hondarribia: Hirú.

Stiegler, B. (2004). La técnica y el tiempo III. El tiempo del cine y la cuestión del malestar (Trad. B. Morales). Hondarribia: Ed. Hirú.

Vinck, D. (2012). Pensar la técnica. Universitas Philosophica, 29(58), 17-37.

Winner, L. (1986). ¿Los artefactos tienen política? En La ballena y el reactor (pp. 2547). Barcelona: Gedisa. 